International Journal of Linguistics, Literature and Translation

ISSN: 2617-0299 (Online); ISSN: 2708-0099 (Print)

DOI: 10.32996/ijltt

Journal Homepage: www.al-kindipublisher.com/index.php/ijltt

\title{
Metafunctions in the Thirukkural: A Systemic Functional Linguistics Analysis
}

\author{
Markandan Rubavathanan 8 (iD. \\ Department of Public Administration, Uva Wellassa University, Badulla 90000, Sri Lanka \\ College of Foreign Languages and Cultures, Xiamen University, Xiamen 361005, China \\ $\triangle$ Corresponding Author: Markandan Rubavathanan, E-mail: ruban@uwu.ac.lk
}

\begin{abstract}
ARTICLE INFORMATION ABSTRACT
Received: May 02, 2021

Accepted: June 14, 2021

Volume: 4

Issue: 6

DOI: 10.32996/ijltt.2021.4.6.1

\section{KEYWORDS}

Thirukkural, Systemic Functional

Linguistics, Thiruvalluvar,

Metafunctions, Tamil

Literature

'Thirukkural' written by Thiruvalluvar, is the most well-known and highly regarded work in the history of Tamil literature. Also, it is well known for its particular structure and wording. It is a distinctive and highly appealing work. Thirukkural has been studied approaching different literary theories. This paper tries to analyse Thirukkural based on the functional method of M. A. K. Halliday, his Systemic Functional Linguistics (SFL). Here, one chapter - Education - of 133 chapters of the Thirukkural is analyzed using Systemic Functional Linguistic framework to achieve functional groupings of the writer's linguistic choices in order to express the meanings and find out how functional features contribute to making the poem potentially. The linguistic data collected from the Thirukkural were analyzed by using the functional analysis method. Consequently, how interpersonal relationships are created within texts, how information is organized in texts and how the ideological positions of writer are implanted in texts were shown and interpreted.
\end{abstract}

\section{Introduction}

Text analysis has been undertaken for a variety of purposes, and in each case, the object of study is always 'the text'. Yet, the shortcoming in most textual analyses is their dependence on the contestable and value judgments of the literary critics, who merely focus on what a text denotes and for whom it suffices to define a text as good or bad by means of implicit assumptions. Such a prescriptive commentary on a text is not instrumental in exploring its multiple meanings. Consequently, linguistics is of prime importance in a textual study, as it explores how language as a system operates in order to create the text by means of linguistic patterns (Fairclough, 2003). Data derived from a linguistic analysis of the text are used primarily to interpret the text at a micro level or the evaluation of the text at a macro level. At the same time, linguistic analysis is not merely concerned with the understanding of the text - what the text means/does or the evaluation of the text as 'good' or 'bad' but rather it deals with the demonstration of how and why a text gets to be the way it is as well as the reader comes to interpret it in a particular way. Therefore, a linguistic analysis of a text involves the explanation of the process whereby the particular meanings of the text are made in the linguistic system and aims at explaining the interpretation and evaluation that are put upon that text because "the role of linguistics is to say how and why the text means what it does to the reader or listener, and how and why he evaluates it in a certain way (Halliday \& Hasan, 1985)".

The linguistic interpretation of the process by which a text is generated necessitates its grammar analysis. The critical role of grammar in textual analyses is also pinpointed by Halliday (1994), a discourse analysis that is not based on grammar is not an analysis at all, but simply a running commentary on a text, for a text, being a semantic unit, embodies meanings that are realized through wordings, and without a theory of wordings - that is, a grammar - there is no way of making explicit one's interpretation of the meaning of a text. In this regard, grammar can be conceived as an instrument for putting the multiple meanings of a text into words. Consequently, "the categories and methods of linguistics enable critics to focus not on the meanings of a work and its implications or value but on the structures that produce meaning during text generation (Hasan, 1989)".

$\begin{array}{lllll}\mathbf{K} & \mathbf{C} & \text { AL-KINDI CENTER } \\ \mathbf{R} & \mathbf{D} & \text { FOR RESEARCH AN }\end{array}$

$\mathbf{R}$ D DEVELOPMENT

Your gateway to world-class research

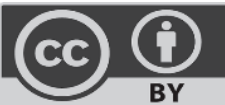

Published by Al-Kindi Center for Research and Development, London, United Kingdom. Copyright (c) the author(s). This open access article is distributed under a Creative Commons Attribution (CC-BY) 4.0 license 
Yet, traditional approaches to the study of literary texts model text analysis as an interpretive activity, where people learn to read a text and try to argue about what meanings they think the writer was making in the text. However, text analysis is not an interpretive but an explanatory activity (Eggins, 2000, p. 309). In fact, the linguistic analysis of the text is not an interpretation of that text; it is an explanation - an explanation of both "WHAT" and "HOW" a text means (Eggins, 2000; Halliday \& Hasan, 1985). In this way, people will become effective readers, who can see the text's contractedness and the reading position imposed upon them by the writer (Kress, 1990).

\section{Theoretical Framework}

In Systemic Functional Linguistics (SFL), language is viewed as a system comprising three layers or strata: a semantic stratum, a lexico-grammatical stratum and a phonological stratum. The semantic stratum accounts for the different facets of meaning in a text: ideational, interpersonal and textual (Halliday, 1987, 1994; Hasan, 2015). These three functional components are also known as metafunctions in the semantic organization of the linguistic system:

i. the ideational metafunction concerns the representation of the speaker's individual experience,

ii. the interpersonal metafunction concerns the expression of the speaker's attitudes,

iii. the textual metafunction concerns the text forming resources of language

Now that these three modes of meaning are present in every use of language in every social context, a text, being a product of all three, can be defined as "a polyphonic composition in which different semantic melodies are interwoven to be realized as integrated lexicogrammatical structures" (Halliday, 1987). In other words, the lexico-grammatical stratum is the level where lexical and grammatical structures which realize the output from the semantic components are mapped onto one another (Morley, 1985). In Halliday's (1987: 134) own words, lexicogrammar "acts as the integrative system, taking configurations from all the components of the semantics and combining them to form multilayered, polyphonic structural positions".

In the conversion of semantic metafunctions into structural patterns, three lexicogrammatical systems of TRANSITIVITY, MOOD and THEME are at work: i. Transitivity involves a ternary configuration of a process, participants and circumstances, ii. Mood realizes the speech functions, iii. Theme concerns how the information within individual clauses is embedded in the larger text (Martin, Matthiessen \& Painter, 1997).

Because every sentence in a text is multifunctional, a person who needs to understand some stretch of language or one who learns through listening or reading will explore these three metafunctions realized through lexicogrammar (Halliday \& Hasan, 1990). Therefore, a clause is a product of three kinds of co-existing structures formed out of elements in Transitivity, mood and Theme systems of lexicogrammar.

In this study, grammar viewed as the foundation stone of textual organization is untraveled for the sake of the narrative exegesis of the Thirukkural. The three research questions of the study can be worded as follows: 1. how are the interpersonal relationships created within the texts? 2. How is information organized in the texts? And 3. How are the ideological positions of the writers implanted in the texts?

\section{Data from Thirukkural}

The Thirukkural (திருக்குறள்) (or the Kural as it is popularly called) is a small collection of 1330 aphorisms, written in metrical verses of two lines and considered as the Bible of South India (Schweitzer, 1957). The Kural is easily the most popular contribution from Tamils to the storehouse of Indian literature. The word "Thirukkural" is composed of two words Thiru and Kural (திரு+குறள்) which mean 'Sacred' and 'Couplet' respectively. The classical literature Thirukkural - Kural was written by Thiruvalluvar (or Valluvar as he is popularly known). Valluvar was a poet, thinker and teacher, all molded into one. According to some scholars, Valluvar lived and wrote during Post Sangam Period, and must have written the Kural sometime between from 300 A.D. to 600 A.D. The Kural, should have existed in written form right from the day of its composition.

Kural stays at the level of general principles, what may be called mandatory ethics? Because Valluvar's message is general, many of his teachings can be applied to varying contexts. Valluvar was not a lawgiver for he was not a prophet. He was a generalist and not a specialist in any particular field. Valluvar never indulged in specifics and he always emphasized the basic principles. Valluvar talks about the responsibilities of individuals, the community and the ruler. He talks about the relationship between a husband and wife, between the king and his people and between the people of the community (Sundaram, 1990; Kulandaisamy, 1994).

Hence, the Kural is a set of 1330 couplets organized in chapters often, each chapter dedicated to a single concept. The concepts are wide, ranging from "Possessing Love", "Truthfulness", "Rain" to "The Avoidance of Tyranny". Each chapter explores different facets of the concept to which it is devoted, starting simply and becoming progressively more complex. There is often a link between chapters where concepts are related: so for example, there are five consecutive chapters on friendship entitled 
"Friendship", "Testing fitness for friendship", "Old friendship", "Harmful friendship" and "False friendship". It is often said of the Kural that there is no aspect of life not covered by it.

Valluvar's couplets reflect the vast amount of knowledge he had in all walks of life: domestic, social, political, and even spiritual. The structural organization of the Kural also reveals much about the values and philosophy of Tamil society (Pillai, 1971). The chapters of ten couplets are grouped into three themes or parts, namely Aram (=Virtue), Porul (=Wealth) and Inbam (=Love). Indeed, Valluvar's Aram, Porul and Inbam are universal in character and secular in nature.

The 3 divisions in the Kural are covered under 133 chapters and each chapter has 10 couplets (133x10=1330, 2660 lines). Aram Virtue 38 chapters, Porul - Wealth 70 chapters and Inbam - Love 25 chapters.

The Kural follows a disciplined poetic structure. Signifying its comprehensive intent, the first couplet begins with the first letter of the Tamil alphabet, $\mathrm{m} \mathrm{A}$ and the last couplet ends with the letter $\mathrm{d}$; $\mathrm{N}$, completing the alphabet. Each couplet consists of seven cirs (சீர்): a cir is a word or combination of words joined together (a compound word) in two lines.

\section{வாய்மை /யெனப்படிவ /தியாதெனின்: யாதொன்றுந் /தீமை /யிலாத /சொலல்}

(Kural: 291)

What is truthfulness? Utterances wholly devoid of ill.

(Here cirs are divided in to 7 units)

A close look at the different chapters would reveal that Valluvar composed every chapter, perhaps in response to a demand or request to produce 10 best couplets on a particular subject. He would bring together all his experience, the knowledge he has gained in mastering other texts and all the information available as folklore in order to compose 10 couplets on a particular subject.

The subject of the research is a written text, a chapter of literature. The data collection will mean selecting relevant extracts from the book Thirukkural. The entire book has 133 chapters with 1330 couplets in all, but it will not be feasible to work on it all because the study is of limited scope. It is not necessary either since choosing a relevant sample can yield the needed data. This study analysis on a selected chapter 'Kalvi - Education' of the second chapter of the division of Porul-wealth it has 10 couplets each has two lines with seven (7) cirs- (a word or a compound word).

\section{Findings and discussions}

The Thirukkural is composed in pure Tamil the language of this Kural differs from the ordinary spoken Tamil. Of this pure and primitive Tamil this work of Thiruvalluvar is the moat finished specimen and may fitly be termed 'a well of Tamil undefiled. The form of the text presents a difficulty to the reader. The greatest difficulty to the reader arises from Kural VeNpa (a Kind of forms to compose a poem), which in all its forms rules over Tamil poetry. The meter requiring much to be compressed into two lines, having seven (7) cirs. This is its difficulty, and this also is one of its great beauties.

In general, finite verb is omitted whenever this is possible. The ordinary forms of the present tense of the finite verb do not occur in the Kural. Besides these, the negative verb, and the imperative, optative, and prohibitive moods, are extensively employed. E. g. Kural 33, 140, 882. Whenever the verb 'to be' is used to complement the predicate, the verb of incomplete predication, or copulative verb, is omitted (Kural 29).

In the Kural there are but two tenses, a past and a future or aorist. The past tense is found (Kural 3, 51, 124, 923), and sometimes is functioning as a predicate. But the future is most often found (Kural 36, 282, 467, 1328). This is used as a true aorist, expressing what belongs to any time or all times. But no example of the present tense is found in the Kural.

Some couplets have no verbs, and they are composed by using noun phrases. In the Kural the noun itself, or its inflexional base, is normally used for every case. E.g.

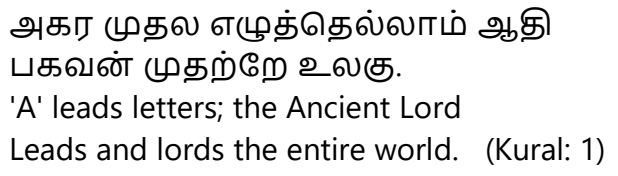

\subsection{Ideational Function}

In the ideational function, the language is carrying the message of embodying the writer to the reader and the speaker to the listener. It does so firstly be sentence patterns and transitivity. In sentence patterns, the clauses are observed and intransitivity of the processes (verbs patterns) are seen. The following tables are showing the transitivity patterns in the chapter 'education'. 


\begin{tabular}{|c|c|c|}
\hline Process & Process word(s) & Participant \\
\hline Material & $\begin{array}{l}\text { 391, } 395 \text { - Katka- learn } \\
391 \text { - nitka - stand } \\
\text { 396 -Oorum- springs } \\
\text { 396- Naadu- learn more } \\
\quad \text { Oor- place }\end{array}$ & you \\
\hline Mental & $\begin{array}{l}394 \text { - uvappa- enjoy } \\
399 \text { - inpuruthal- joy }\end{array}$ & Scholar, intellectuals \\
\hline Relational & 393 & educated and uneducated \\
\hline \multicolumn{3}{|l|}{ Verbal } \\
\hline \multicolumn{3}{|l|}{ Behavioral } \\
\hline Existential & $\begin{array}{l}392 \text { - enpa - are } \\
398 \text { - udattu - have }\end{array}$ & $\begin{array}{l}\text { Letters and Numbers } \\
\text { the one }\end{array}$ \\
\hline
\end{tabular}

From this table, it is noticed that the material process is used most in the chapter to show the action that consists of around 50 percent. As it is well-known that the material process shows "doing" so the description of such things in the poem reveals that under discussion thing/object or agent is active to do and leave some effects on the goals.

On the other hand, second, most used process is mental. In poetry, this kind of description is vital to have emotional or sentimental effects. Then there are existential processes. And finally verbal, and behavioural processes are not found in the poem.

\subsection{Interpersonal Function}

This category of grammatical analysis of the systemic functional grammar is to analyze the interacting elements in the text. Its approach is entwined with discourse analysis and its speech act theory. "Halliday approaches the grammar of interaction from a semantics perspective. He points out that whenever we use language to interact, one of the things we are doing with it is establishing a relationship between us" (Eggins, 2000). In that perspective following is the analysis of the relationship of the factors.

\begin{tabular}{|l|l|}
\hline Personal pronouns type & Personal pronoun \\
\hline Second Person & Nee - You \\
\hline Third Person & Avarkal - They \\
& Oruvar - The one \\
& Thaam - They \\
& Pulavar - Scholar \\
& Kattaar - Educator \\
& \\
\hline Third Person non-human & Arivu - knowledge \\
& Kalvi - Education \\
\hline
\end{tabular}

From the table, it can be observed the participants in the chapter are mostly the third person. It is because the poet embodies the readers with another person. There is no first-person pronoun in this chapter. Third-person pronoun is given not for a reader-writer gap but here third is used to describe the things or phenomena that are the same from the readers and the poet. And there is no second person to whom the poet may address or tell something him as the second person considering it as a separate entity from him.

\subsection{Textual Function}

The textual function is aimed at the realization that the language has in the way of coherent mechanism, written or spoken discourse, rather than random selection of sentences. It also makes the written or spoken discourse a living piece of writing and a unified text that offers logic and thoughtful. In it, the sentence structure or specifically "theme" and "rheme" is analyzed in order to know that what is said to whom.

From this point of view, it can be seen that the poem is mostly about the objects which are a doer or described rather than the observer or the poet who is walking over there such as: 
E.g. Kural 393

Kannudaiyaar enpavar kattor

Men who have eyes/ are educators

Kannudaiyaar(Theam) enpavar kattor (rheme)

The above example of theme/rhyme description shows that it is a very objective poem in which doers or action-oriented things are objects. Before doing any action, the reader or poet is already done by the object described in the poem.

\section{Conclusion}

The main objective of this study is to analyze Thirukkural in the theory and framework of Systemic Functional Linguistics (SFL). In this study, SFL theory and analysis are approached from a metafunctional analysis and lexicogrammatical perspective that views language usage in a text from one of the chapters Kalvi -Education of Thirukkural multi-layered system that configures and expresses the meanings in relation to the context of use. SFL offers a comprehensive and systemic model of language that permits language patterns to be described at different levels. It theorizes the relations between language as a social semiotic so that texts (Kurals) from Thirukkural can be seen as reproducing of interpersonal relations, ideational functions and textual organizations. Accordingly, the way in which the method of metafunctional analysis applied in this study has shown its meaning potential of Thirukkural through the paradigmatic and syntagmatic axis of lexicogrammar and their functions.

Indeed, it is to note that the interpretation of this metafunctional approach to the text extracted from Thirukkural can become an indispensable analysis for the entry of teachers in mandatory Tamil language and literature classrooms and even for creative writers who place themselves in textual criticism. At the same time, this study's outcomes support other existing research findings in the field of SFL text analysis. SFL as a field of research method could benefit from theoretical and practical application insights from these kinds of multilingual text analysis and future research directions.

\section{References}

[1] Eggins, S. (2000). An Introduction to Systemic Functional Linguistics. London: Pinter Publishers.

[2] Fairclough, N. (2003). Analyzing discourse: Textual analysis for social research. London: Routledge

[3] Halliday, M. A. K. (1987). Language as Social Semiotic. London: Edward Arnold.

[4] Halliday, M. A. K. (1994). An Introduction to Functional Grammar. London: Edward Arnold.

[5] Halliday, M. A. K. and Hasan, R. (1985). Cohesion in English. New York: Longman Inc.

[6] Halliday, M. A. K. and Hasan, R. (1989). Language, Context, and Text: Aspects of Language in a Social-Semiotic Perspective. Hong Kong: Oxford University Press.

[7] Hasan, R. (1989). Linguistics, Language, and Verbal Art. Hong Kong: Oxford University Press.

[8] Kress, G. (1990). Linguistic Processes in Sociocultural Practice. Hong Kong: Oxford University Press.

[9] Hasan, R. (2015). Choice, system, realization: Describing language as meaning potential. In L. Fontaine, T. Bartlett, \& G. O'Grady (Eds.). Systemic functional linguistics: Exploring choice (pp. 269-299). Cambridge: Cambridge University Press.

[10] Kulandaisamy, V. C. (1994). The immortal Kural. New Delhi: Sahitya Academy.

[11] Martin, J. R., Matthiessen, C. M. I. M. \& Painter, C. (1997). Working with Functional Grammar. London: Arnold.

[12] Pillai, M.S. (1971). A study of Prosody and Various readings in Thirukkural. Madras: University of Madras.

[13] Schweitzer, A. (1957). Indian thought and its development. London: The Beacon Press.

[14] Sundaram, P.S. (Trans. \& Ed.) (1990). The Kural. London: Penguin Classics \& Penguin Books 


\section{Appendix}

திருக்குறள் - பொருள் - அரசியல் - கல்வி - அதிகாரம் 40

\section{Thirukkural - wealth - Royalty - Education - Chapter 40}

கல்வி EDUCATION

391. கற்க கசடறக் கற்பவை கற்றபின் நிற்க அதற்குத் தக.

Lore worth learning, learn flawlessly Live by that learning thoroughly.

392. எண்ணென்ப ஏனை எழுத்தென்ப இவ்விரண்டும் கண்ணென்ப வாழும் உயிர்க்கு.

Letter, number, art and science

Of living kind both are the eyes.

393. கண்ணுடையர் என்பவர் கற்றோர் முகத்திரண்டு புண்ணுடையர் கல்லா தவர்

The learned alone have eyes on face

The ignorant two sores of disgrace.

394. உவப்பத் தலைக்கூடி உள்ளப் பிரிதல் அனைத்தே புலவர் தொழில்.

To meet with joy and part with thought

Of learned men this is the art.

395. உடையார்முன் இல்லார்போல் ஏக்கற்றுங் கற்றார் கடையரே கல்லா தவர்.

Like poor before rich they yearn:

For knowledge: the low never learn.

396. தொட்டனைத் தூறும் மணற்கேணி மாந்தர்க்குக் கற்றனைத் தூறும் அறிவு. As deep you dig the sand spring flows As deep you learn the knowledge grows.

397. யாதானும் நாடாமால் ஊராமால் என்னொருவன் சாந்துணையுங் கல்லாத வாறு.

All lands and towns are learner's own

Why not till death learning go on!

398. ஒருமைக்கண் தான்கற்ற கல்வி ஒருவற்கு எழுமையும் ஏமாப் புடைத்து.

The joy of learning in one birth

Exalts man up to his seventh.

399 தாமின் புறுவது உலகின் புறக்கண்டு காமுறுவர் கற்றறிந் தார்.

The learned foster learning more

400. கேடில் விழுச்செல்வம் கல்வி யொருவற்கு மாடல்ல மற்றை யவை.

Learning is wealth none could destroy 400 Nothing else gives genuine joy. 